\title{
Alzheimer Disease Detection Techniques and Methods: A Review
}

\author{
Sitara Afzal ${ }^{1}$, Muazzam Maqsood ${ }^{1 *}$, Umair Khan ${ }^{1}$, Irfan Mehmood², Hina Nawaz ${ }^{1}$, Farhan Aadil ${ }^{1}$, Oh-Young \\ Song ${ }^{3 *}$, Yunyoung $\mathrm{Nam}^{4 *}$
}

${ }^{1}$ Department of Computer Science, COMSATS University Islamabad, Attock Campus (Pakistan)

${ }^{2}$ Department of Media Design and Technology, University of Bradford, Bradford (UK)

${ }^{3}$ Department of Software, Sejong University, Seoul 05006 (Korea)

${ }^{4}$ Department of Computer Science and Engineering, Soonchunhyang University, Asan 31538 (Korea)

Received 25 August 2020 | Accepted 17 October 2020 | Published 21 April 2021

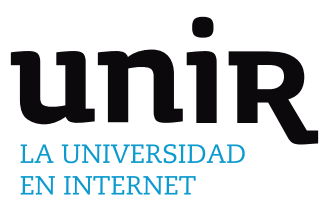

\section{ABSTRACT}

Brain pathological changes linked with Alzheimer's disease $(\mathrm{AD})$ can be measured with Neuroimaging. In the past few years, these measures are rapidly integrated into the signatures of Alzheimer disease (AD) with the help of classification frameworks which are offering tools for diagnosis and prognosis. Here is the review study of Alzheimer's disease based on Neuroimaging and cognitive impairment classification. This work is a systematic review for the published work in the field of $\mathrm{AD}$ especially the computer-aided diagnosis. The imaging modalities include 1) Magnetic resonance imaging (MRI) 2) Functional MRI (fMRI) 3) Diffusion tensor imaging 4) Positron emission tomography (PET) and 5) amyloid-PET. The study revealed that the classification criterion based on the features shows promising results to diagnose the disease and helps in clinical progression. The most widely used machine learning classifiers for AD diagnosis include Support Vector Machine, Bayesian Classifiers, Linear Discriminant Analysis, and K-Nearest Neighbor along with Deep learning. The study revealed that the deep learning techniques and support vector machine give higher accuracies in the identification of Alzheimer's disease. The possible challenges along with future directions are also discussed in the paper.

\section{KEYWORDS}

\author{
Alzheimer's Disease \\ Review, Mild \\ Cognitive Impairment, \\ Neuroimaging, Machine \\ Learning Classifiers, \\ Deep Learning.
}

\section{INTRODUCTION}

\begin{abstract}
$\mathrm{T}$ HE most frequent form of dementia is Alzheimer's disease. About 1 out of 85 people in the world are suspected to have Alzheimer's Disease by 2050 [1]. In this disease, neurons are lost because of the accumulation of abnormal proteins in the form of plaques tau tangles of neuro-fibrillary in the brain of a person [1]. AD occurs in the temporal lobe of the brain and hippocampus [1] thus changes the brain even before the symptoms of dementia occur [2]. It has been proposed that this inescapable decay can be a profitable marker of neurodegeneration, as estimated with sMRI (Structural Magnetic Resonance Imaging). Moreover, functional MRI (fMRI) and fluorodeoxyglucose positronemission tomography (FDG-PET) [3], [4] detect the alterations in function, metabolism, and connectivity. In the starting stages of $\mathrm{AD}$, it becomes hard to distinguish between the patterns with quantitative analysis and even with radiological readings, this is because of the nuance in the pattern. So, it is challenging to diagnose and monitor disease at its early stage.
\end{abstract}

Individuals in the underlying phase of Alzheimer's Disease are

* Corresponding author.

E-mail addresses: muazzam.maqsood@cuiatk.edu.pk (M. Maqsood), oysong@sejong.edu (O. Y. Song), ynam@sch.ac.kr (Y. Nam). considered to have Mild-Cognitive-Impairment (MCI) [5], even though not every individual grows Alzheimer's Disease having MCI. Mild-Cognitive-Impairment is a provisional phase from typical to Alzheimer's Disease, where an individual has gentle changes in the psychological capacity, which is evident to the individual and family members, yet the individual can perform daily routine activities.

Around 15 to almost $20 \%$ of individuals, matured to $60+$ or more have Mild-Cognitive-Impairment, and 30 to almost $35 \%$ of people with $\mathrm{MCI}$ grow to $\mathrm{AD}$ within 4 years [6]. This transition takes time between six to three years, yet ordinarily, it takes a year and a half. MCI patients would then be able to be classified as Mild-CognitiveImpairment converters or non-converters; means that the individual may or may not have changed to $\mathrm{AD}$ within a year and a half. There are additionally different subtypes of MCI that are occasionally referenced in the writing, for example, first MCI. The utmost critical hazard influences for Alzheimer's Disease are family ancestries as well as the nearness of linked qualities in an individual's genes. An Alzheimer's Disease evaluation depends on a medical assessment, just as an exhaustive meeting of the patient and their family members [7]. The evaluation of Alzheimer's Disease must be completed using dissection, which is not clinically useful [8]. Without this groundtruth information, individuals require additional models to diagnose Alzheimer's Disease. These measures can enhance the comprehension of $\mathrm{AD}$, and make analysis workable for existing individuals. 


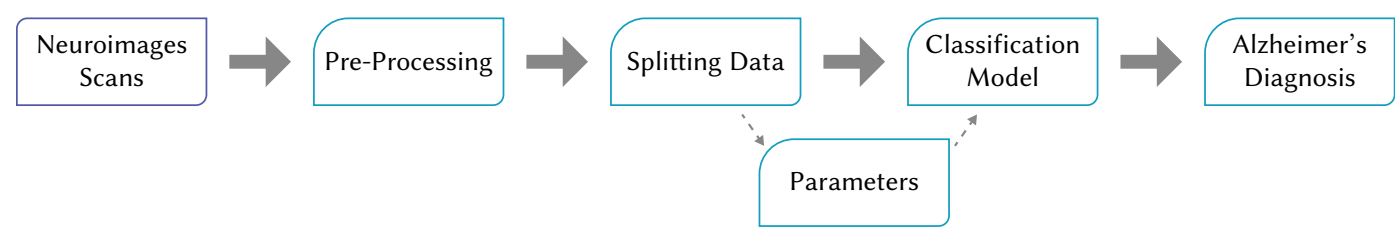

Fig. 1. The general flow of Computer-Aided Diagnosis (CAD) system in the diagnosis of Alzheimer's.

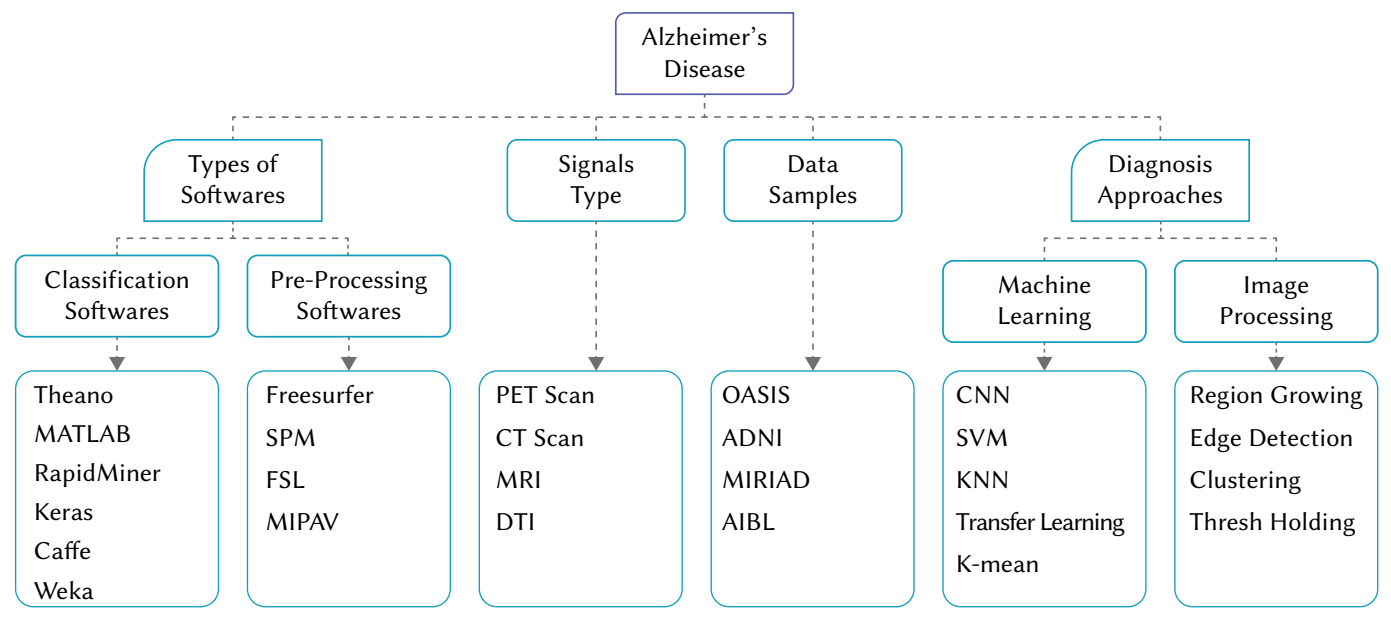

Fig. 2. The overall flow diagram of the survey.

Various neuroimaging studies, which have used region of interest (RoI) to find out the subtle changes related to $\mathrm{AD}$, were solely dependent on previous knowledge to suggest the selection of features, ignoring the changes in the brain besides the region studied. Fig 1 demonstrates the general flow of the CAD system in the diagnosis of Alzheimer's. In contrast, machine learning is a systematic approach that develops automatic as well as objective classification and analyzes huge amounts of data either complex or simple and efficiently distinguishes between the subtle changes occurring in the brain images.

Mostly, feature extraction and classification methods, i.e.; classification frameworks make predictive models [9] to help in decision making and facilitating automation of medical decisions. Moreover, imaging markers or records can be created by using classification frameworks with improved affectability and an individual's specificity. This makes a more individualized and tolerant customized approach, which is basic in the present period of customized medication. It permits to encourage the thought of hereditary or way of life dangers, by using progressed computational control. In the past decades, a lot of work has been done on the neuroimaging-based classification of $\mathrm{AD}$, to make it computerized to diagnose at its early stages. Its rapid study has motivated us to summarize different AD-related work from feature extraction using different neuroimaging data to different classification methods. Moreover, we discuss problems related to the limited sample size and data setting variability by analyzing different studies. The most significant and primary test in $\mathrm{AD}$ appraisal is to decide if somebody has Mild-Cognitive-Impairment and to anticipate if a MildCognitive-Impairment individual will build up into neurodegenerative sickness. Different phases of the disease, for example, early/late MCI are equally important to diagnose. Identifying $\mathrm{AD}$ utilizing Deep learning is generally a test for analysts due to:

- Poor quality images during the pre-processing phase.

- Unavailability of publicly available large data samples for research.

- Limited labeled dataset for AD.

- Deficiency of essential data points, specifically for the identification of ROI in the cerebrum.
The general flow of the $\mathrm{CAD}$ system in the diagnosis of Alzheimer's is demonstrated in Fig. 1. We will generalize the existing $\mathrm{AD}$ classification studies. Furthermore, critical parts, which were previously not explored in $\mathrm{AD}$ will also be discussed. The past review papers on $\mathrm{AD}$ [4], [10], limited $\mathrm{AD}$ classification to MRI but the pathological changes in the brain-related to $\mathrm{AD}$ can be diagnosed by several different modalities of imaging which include FDG-PET and amyloid-PET. This is the reason that a comprehensive review of $\mathrm{AD}$ classification is needed.

This paper also discusses cross-validation strategies i.e. independent training and testing of classification algorithms. It uses two strategies, split-n-train and k-fold cross-validation [11], [12] for unbiased results. We further discuss the different imaging modalities used for $\mathrm{AD}$ identification along with machine learning techniques and algorithms used in this domain. We conclude our review by highlighting the limitations and research challenges along with possible future research directions for researchers.

\section{SCOPE OF THIS REviEW}

Computer-Aided-Diagnosis (CAD) of Alzheimer's has opened an important area for the early detection of Alzheimer's Disease. In this survey, the papers are reviewed from repositories of IEEE, ACM libraries, Science Direct, Springer containing keywords like AD, Alzheimer's Disease, deep learning, machine learning, and image processing. These all online databases were selected as they are wellknown for their authenticity and they offered the most significant peer-reviewed articles covering the field of image processing, machine learning, and deep learning. While going through these databases, the terms used for searching was expecting to cover most of the effort including image processing, neural networks, and machine learning approaches for the identification of Alzheimer's Disease. The scope of the paper is to make a survey and analyze the studies and research of different groups in image processing-based approaches as well as machine learning-based techniques. This paper also helps the new researcher who is starting to explore the computer-aided methods for $\mathrm{AD}$ diagnosis. Fig. 2 shows a general overview of this paper. 
III. IMAGING MODALITIES

Different enhanced imaging techniques are being used to identify the signals that are leading toward more accurate AD detection. The quantitative analysis of brain degeneration identification is being more comprehensively applied. Diverse neuroimaging-based signals like CT scan, PET, sMRI, fMRI, and DTI are utilized to produce a more conclusive prediction.

\section{A. Computerized Tomography (CT) Scan}

CT scan is a cross-sectional illustration of the brain region that is produced with the help of an x-ray with a constant bombardment of radioactive rays. These images are $100 \%$ more transparent than normal $\mathrm{x}$-rays. CT scans cannot be considered as a benchmark for the early detection of $\mathrm{AD}$ as other advanced methods are providing precise and accurate results. Some studies tried to endorse its efficiency in affected $\mathrm{AD}$ detection by emphasizing its coherence and cost-effective conclusions in comparison with other techniques like PET or MRI. It is safe to conclude that it does not play any substantial role in the early detection of $\mathrm{AD}$.

\section{B. Positron Emission Tomography (PET)}

PET scan is a volumetric subatomic illustration method that is used to obtain a 3D brain scan on anatomical and sub-anatomical levels.

PET scanning is done by administering or inhaling a radioactive isotope as a tracing agent also known as a radiotracer. That works as a positron-emitting spec for the subject. Then this radiotracer is detected by a scanning machine. Afterward, the scanner provides a digital image (illustration) of the radiotracer spread in the subject body. The nature of the PET scan depends on a different kind of radiotracer being used. The cost of PET scanning has been raised due to the use of cyclotron agents that play an essential role in the preparation of radiotracer. As the brain functionality depends on the consumption of blood sugar, so the illustration can be deduced that glucose consumption and neural functioning are directly proportional. The PET scan is very peculiar in the prediction of $\mathrm{AD}$ even with mild symptoms. The working of PET scanning is quite effective, but the reasons mentioned above show that this is not a healthy diagnostic method.

\section{Structural Magnetic Resonance Imaging (sMRI)}

MRI is a non-invasive (non-anatomical) imaging (illustration) method that is used for structural evaluation of the concerned brain region. MRI scanners are used in brain scanning. The MRI procedure involves the bombardment of the magnetic rays on the area of which imaging is required. Different kinds of areas are identified due to tissue movement. MRI is widely utilized for the early diagnosis of $\mathrm{AD}$. The number of studies based on MRI has been increased for years due to the availability of open-source databases like ADNI and OASIS. This has also impacted the studies driven around these databases to help detect disease progression monitoring and improved analytical studies. With the help of MRI, the disease impact on the subject's spatial domain and temporal region creating patterns can also be observed. MRI-based studies helped to verify results that the brain tissue degeneration in patients affected with $\mathrm{AD}$ and transitioning from $\mathrm{MCI}$ to $\mathrm{AD}$ can be a speedier process than that of a healthy individual. The concerned domain in this regard could be to detect subjects with $\mathrm{MCI}$ which can be done with early detection of $\mathrm{AD}$. The accuracy for MRI based model is $93.18 \%$ with $93 \%$ precision, $92 \%$ recall, and $92 \%$ f1-score. Various studies show that early detection of AD with the help of MRI is not $100 \%$ accurate. The degeneration in the hippocampal region can easily be identified in a patient suffering from $\mathrm{AD}$ in comparison with a non- $\mathrm{AD}$ patient. As in many cases, the brain damage is solely not due to $\mathrm{AD}$ so determining these meek differences can be critical. But over time MRI based studies (automatic MRI) showed promising improvement. Currently, MRI-based techniques are widely used for the early detection of AD because MRI devices are easily available now.

\section{Functional Magnetic Resonance Imaging (fMRI)}

The process of functional MRI is also a non-invasive procedure that aids the diagnosis of malfunction caused by AD. fMRI also allows observing the absorption of oxygen during resting and active state to form an activity pattern. So, brain activity during different states can be evaluated. fMRI extracts data from each region of the brain to help diagnose AD. Studies over the period show that patients suffering from $\mathrm{AD}$ have reduced activity in the limbic region especially in the hippocampus due to brain damage, and plaque abnormalities, and cerebral cortex region due to vascular damage. But these exceptions are less notable in patients suffering from MCI that indicate the less evident use of fMRI in early detection of MCI. Some studies have also shown some incongruous conclusions for hippocampus regions. In this regard, a resultant U-curve shape is formed. One of the most beneficial uses of fMRI is that it does not involve the use of radioactive substances or radiations due to this fMRI can be used as many times as needed. As patients advance levels of disease, suffering from extreme cognitive impairment, they cannot have adequate motor control. So, to get good results patients need to be steady while going through the scanning process. The prompt changes of $\mathrm{AD}$ are due to the neuro-degeneration process of intentional availability between different brain regions [13]. Different researchers working on rsfMRI have talked about the presence of common changes inside the gut concerning resting-state systems. Oxygen level-subordinate that are initiated as local patterns are generally concerned for the most stressed part of cerebrum capacities like tangible, and non-appearance color innovation [14] [15] [16] [17] [18] [19].

\section{E. Diffusion Tensor (DTI)}

DTI is an MRI-based imaging technique that illustrates minute structural cross-sectional details of brain regions. These samples are also procured non-invasively with MRI scanners. DTI is roughly based on the Browning motion sensation of water molecule activity of human tissues. So, this phenomenon can be described as the microscopic dimension that measures the size, dimensionality, and orientation of the tissue that helps identify the last stage of microscopic degeneration. Studies show that DTI can be an implicit mechanism to aid early-stage $\mathrm{AD}$ identification. The Look into utilizing DTI-based features, might lie in expansion isolated under three categories, contingent upon how characteristic would be extracted: i) tractography, ii) integrated network measure process, then iii) distinctive voxel-preference approach.

In the respective section, different types of images utilized in the detection and classification of $\mathrm{AD}$ along with their modalities were discussed. Their utilization in the existing literature was also referenced and elaborated. Fig. 3 summarizes the modality of images through the modality chart of images utilized in the above-mentioned techniques.

IV. State of the Art Alzheimer's Detection AND Classification Methodology

\section{A. Image Preprocessing}

Multiple approaches to image processing were deployed for the diverse domain of studies. In [8] the image processing process involved visual inspection for essential irregularities distinct to Alzheimer's or FTLD and artifacts. The method included: the use of SPM5 (http:// www.fil.ion.ucl.ac.uk/spm); The combined images of groups I and III and patients from II and IV using in implementation diffeomorphic 


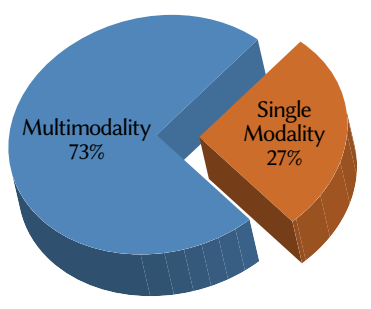

a

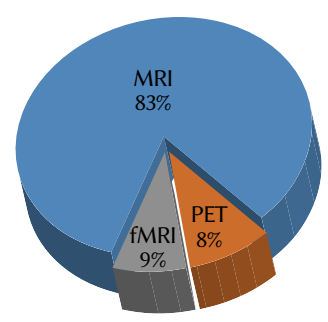

$\mathrm{b}$

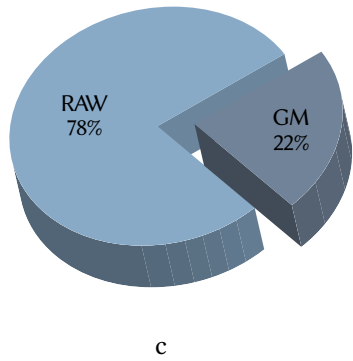

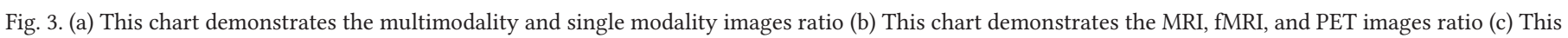
chart demonstrates the RAW and Gray-Matrix (GM) ratio.

TABLE I. Feature Extraction Techniques Using Machine Learning

\begin{tabular}{|c|c|c|}
\hline Type & Description & Benefits \& Limitations \\
\hline $\begin{array}{l}\text { Local Binary } \\
\text { Pattern (LBP): }\end{array}$ & $\begin{array}{l}\text { The working of LBP is by limiting a certain gap of image pixels utilizing the middle } \\
\text { image pixel value and understands output as a dual array i.e. binary pattern. These } \\
\text { image pixels are further labeled by utilizing these patterns. The histogram of these } \\
\text { arrays signifies the feature description. To create the system, a well-organized } \\
\text { bigger window is suitable, as the values of pixels differ slowly. }\end{array}$ & $\begin{array}{l}\text { Benefits: LBP features are computationally } \\
\text { simple and fast. It also takes a shorter training } \\
\text { time. } \\
\text { Limitations: LBP features are less accurate } \\
\text { because of the high false-positive rate. }\end{array}$ \\
\hline $\begin{array}{l}\text { Scale Invariant } \\
\text { Feature } \\
\text { Transform } \\
\text { (SIFT): }\end{array}$ & $\begin{array}{l}\text { This approach joins identification and explanation of the features [40]. Its working } \\
\text { is by increasing the regions for interest point assortment and further pinpointing } \\
\text { important facts in that picture. The bearings info is formerly given to certain } \\
\text { important points, that in conclusion defines the Scale Invariant Feature Transform } \\
\text { (SIFT). }\end{array}$ & $\begin{array}{l}\text { Benefits: The implementation of SIFT is fast } \\
\text { and has a robust high rate matching. } \\
\text { Limitations: SIFT feature extractions are too } \\
\text { sensitive to input type and smoothing. }\end{array}$ \\
\hline $\begin{array}{l}\text { Gray-Level- } \\
\text { Cooccurrence- } \\
\text { Matrix (GLCM) }\end{array}$ & $\begin{array}{l}\text { It was initially applied for texture examination and it exceeded the best-in-class } \\
\text { methods. The working is stipulating the co-occurrence of different levels of gray at } \\
\text { a precise position in the image in a precise section of the usual image of gray level, } \\
\text { the pixels are generally of related gray level in a precise section, and are greatly } \\
\text { related. }\end{array}$ & $\begin{array}{l}\text { Benefits: The computational time of GLCM } \\
\text { features is low and that is why memory } \\
\text { consumption is also very low. } \\
\text { Limitations: It only works with grayscale } \\
\text { images. }\end{array}$ \\
\hline
\end{tabular}

registration algorithm; The preprocessing steps for [20] for baseline $1.5 \mathrm{t}$ and $\mathrm{T} 1$ weighted MRI dataset from the ADNI repository. These images were normalized and segmented with Statistical Parametric Mapping (SPM) software package. The preprocessing of [21] was conducted with the statistical software package SPM2 (Welcome Trust Centre for Neuroimaging, London, https://www.fil.ion.ucl. ac.uk/spm/). This domain dataset area is of binary class type as they were of Alzheimer's disease (AD) and normal class (NC). Each MRI was $3 \mathrm{D}$, with the tensor intensity value equal to $110 \times 110 \times 110$. As one subject can have more than one MRI in the data repository, to avoid the redundancy between the training and testing dataset option,s the best approach of only using the best and earlier acquired MRI's for individual subjects was considered. For this purpose, multiple diverse amounts of images were selected for each training and testing batch for individual studies. In each study preprocessing method was quite like the MRI's or PET scans however, dimensions were altered according to the dimension requirements of the said algorithms.

\section{B. Image Processing Based Techniques for Alzheimer Detection}

Image processing-based approaches are utilized to extract the features from the images. The following are the different Image processing-based approaches. Table I demonstrates the features that are mostly extracted by utilizing these approaches from neuroimages.

\section{Thresholding}

For image segmentation and object detection, thresholding is one of the most widely used techniques. Thresholding is used to separate and elaborate the concerned terrain of the foreground from the background aptly for analytical purposes. The building block of this method depends on the pixel's intensity values of the image under analysis. Threshold values are specified/classified as an intensity histogram for the background (Erosion) and foreground (Dilation) and then both distinct values are analyzed to separate them.

\section{Region-Based Methods}

In this method, the concerning area is selected based on pre-defined rules according to the nature of the algorithm and dataset. The focus in this regard is the intensity values of the object boundaries in an image. In this process, a seed point is selected and pixels with similar values are used based on properties such as intensity, texture, and color. With these values, concerned regions are separated from the rest of the image. Using the region growing method solely for object detection is not enough as it is usually used with additional techniques like Region splitting, split and merge, and Edge detection.

\section{Clustering}

Grouping together similar data-points is known as clustering. Clustering is an unsupervised learning approach as it is not based on advanced learning of training data.

But the iterative nature of the clustering method takes care of the segmentation method. The process of clustering continues till each similar object is not assigned to a cluster with the same attributes, for this purpose a similarity measure is defined in advance.

\section{Atlas Guided Approaches}

This map or atlas-based approach is used to form an image analysis. The image is designating a specific region to form a shape. The primary motivation behind this methodology is to assist radiologists in the revelation and ID of illnesses. The working progress of the approach is enhanced by distinguishing noteworthy life systems in the clinical images. 


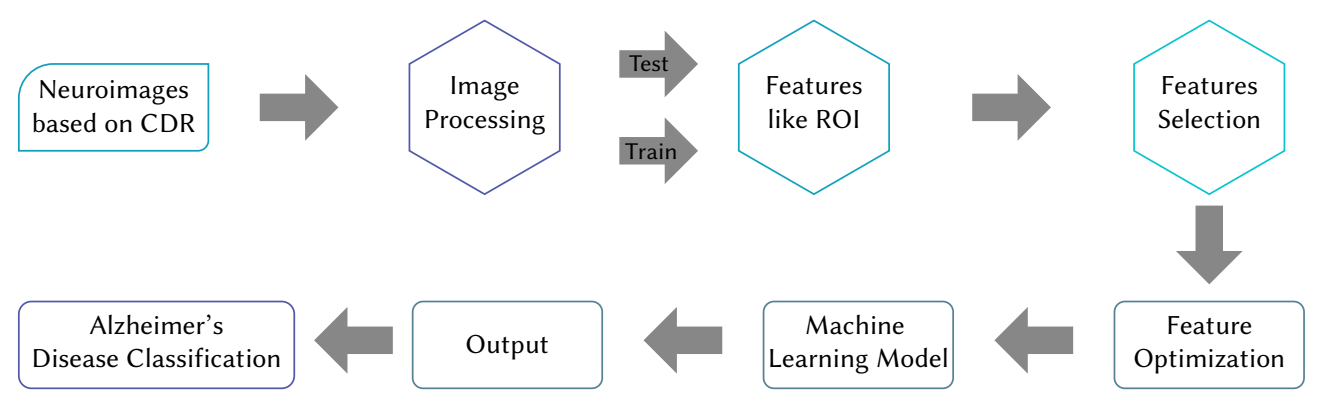

Fig. 4. The fundamental framework for the ML approach for the classification of $\mathrm{AD}$.

\section{Edge-based Methods}

These approaches are used to detect object boundaries. It is also used to find cracks in detected boundaries. This is one of the most widely used methods to detect object boundaries with the same pixel intensities. The distinction between the pixels, in this case, is carried out by estimating the intensity gradient. These methods are mainly used as a base or central technique for other segmentation approaches.

\section{Machine Learning Techniques for Alzheimer Detection}

These techniques are extensively used in the clinical application[22] and also established noteworthy attention in the past eras [23]. It is well-thought-out as a division of $\mathrm{AI}$ as it allows the removal of an expressive model or pattern from samples. These approaches are mainly categorized into clustering/unsupervised and classification/ supervised. Below is a brief explanation of Classification i.e. Supervised Learning and clustering i.e. Unsupervised Learning. The main idea behind this machine learning approach is centered on a trainer which trains label data with the label group by utilizing a training set. Different types of biomarkers are called features that must be learned for the difficulty at hand. Fig. 4 shows the fundamental framework for the ML approach for the classification of $\mathrm{AD}$.

\section{Bayesian Classifiers}

Bayesian classifiers are the simple statistical classifier centered on Bayes theorem with robust (naïve) independence expectations amongst the feature [24]. Instead of undergoing training by a single algorithmic approach, Naïve Bayes learns by utilizing algorithms centered on universal rules. In [25] Seixas et al. give a Bayesian classifier i.e. Bayesian network decision model for helping detection of Alzheimer disease, Mild Cognitive Impairment (MCI), and Normal Control (NC), while they achieved higher results as compared to numerous famous classifiers like simple Naïve Bayes, Logistic Regression Classification (LRC), multilayer perception Artificial Neural Network, decision table, choice base enhanced through Adaboost approach and J48 Decision Tree. In [26] Liu et al. give the multifold Bayesian kernelization approach that could discriminate Alzheimer's disease and normal control with higher accuracy value nonetheless attained bad output in identifying MCI-converter (MCI) and MCI non-converter (MCI). In [27] plant et al. join a particular feature with grouping by utilizing a Bayesian classifier for the judgment amongst Alzheimer's Disease and Normal Control on Magnetic Resonance Images data and stated up to $92.0 \%$ accuracy. In [28] Lopez et al. use the multivariate approach, for example, PCA and Linear Discriminate Analysis for extracting feature, after that applied the Bayesian frame for automatic detection of Alzheimer's Disease and Normal Control by utilizing Positron Emission Tomography i.e. PET and Single Photon Emission Computed Tomography i.e. SPECT.

\section{Support Vector Machine}

SVM i.e. Support Vector Machine is a very common machine learning algorithm used for both regression and classification-based problems.
For non-linear separable data, sample points are mapped over a high dimensional plane exploiting the boundary among points in a high dimensional space [29], [30]. This transformation of data-points from one dimension to another dimensional space is known as the kernel trick [31]. This optimal algorithm is established over the 'Training Set' where the training data is utilized for maturing the procedure that can distinguish among previously defined clusters and a 'Testing Set' where the procedure is utilized to forecast the clusters where the new observations go. To plot the training set from input space to a high-level feature space, Kernel functions can be utilized [32]. The data points that are positioned nearest to the decision surface are support vectors. Support vector machine have different variants, for example hierarchal SVM [33], radial basis function-based SVM [34] and AdaBoostSVM [35]. In the past decades, the interest in SVM in Alzheimer's Disease studies has been increasing [36]. For the classification of patients with Alzheimer's Disease, the early applications of support vector machines to neuroimaging data were mainly meant to translating the mental state of Normal or healthy subjects [37]. Effectively established that it was achievable to differentiate amongst subjects giving correct and incorrect responses with the accuracy of $99.3 \%$ centered exclusively on discriminative forms of brain actions. Recently in 2019, Afzal. S et al. [38] utilized machine learning, the Random Forest approach for the classification of multi-class Alzheimer's Disease, and 92.4\% accuracy was attained in their findings. MicroRNAs or MiRNA are singlestranded non-coding RNA particles that exhibit distinctive expression to varying pathological and physiological conditions. To differentiate between $\mathrm{AD}$ and other neurological ailments that are centered on such genome-based biomarkers, SVM was utilized by Smith-Vikos and Slack [39]. By utilizing three blood-based biomarkers [41], oxidized LDL, antibodies [42], Laske et al. [43] also utilized Support Vector Machine to distinguished patients with Alzheimer's Disease from other normal subjects and attained $81.7 \%$. Higher accuracy was reported by comparing the output of the thresholding-based approach with the SVM-based segmentation. Accuracy of $92.31 \%$ plus $96.67 \%$ was reported for Alzheimer's disease (AD) diagnosis by utilizing Support Vector Machine in PET and SPECT respectively by Lopez et al. [44]. Juergen Dukart et al. utilized SVM to combine magnetic resonance images (MRI) and FDG-PET for enhancing the identification of AD. They casually removed FDG_PET and Magnetic resonance Images from two different databases i.e. ADNI and Leipzing Cohorts. They attained accuracy of $88 \%$ for ADNI datasets and up to $100 \%$ for Leipzing datasets [45]. Y. Zhang et al. distinguish among elderly subjects with Alzheimer's Disease, MCI, and Normal control (NC). They utilized 5-fold cross-validation for KSVM-DT and attained $80 \%$ classification accuracy [46]. P. Padilla et al. proposed a new Computer-Aided Technique (CAD) centered on non-negative matrix factorization and support vector machine for initial $\mathrm{AD}$ analysis. They utilized two databases (PET and SPECT), both databases containing $\mathrm{AD}$ patients and healthy control. They proposed NMF-SVM and yields $91 \%$ accuracy [47]. In [48], researchers used SVM on MRI and SPECT images and attained much higher accuracy for MRI instead 
TABLE II. SVM BASED TECHNIQUES

\begin{tabular}{|c|c|c|c|c|}
\hline Author & Targets & Methods & Imaging Modality & Accuracy \\
\hline Afzal. S et al. [38] & Multiclass AD classification & SVM & MRI & $92.4 \%$ \\
\hline Smith Vikos et al.[42] & $\mathrm{AD}$ vs $\mathrm{NC}$ & SVM & MRI & $90.3 \%$ \\
\hline Laske et al.[43] & $\mathrm{AD}$ vs $\mathrm{NC}$ & SVM & MRI & $81.7 \%$ \\
\hline Lopez et al.[44] & $\mathrm{AD}$ vs $\mathrm{NC}$ & SVM & MRI & $96 \%$ \\
\hline Y.Zhang et al.[46] & $\mathrm{MCI}$ vs NC & SVM & MRI & $80 \%$ \\
\hline P.Padilla et al.[47] & $\mathrm{AD}$ vs $\mathrm{NC}$ & NMF-SVM & PET and SPECT & $91 \%$ \\
\hline Luiz K Feriera et al.[48] & FDG-PERT vs MRI & SVM & PET & $68-71 \%$ \\
\hline \multirow{2}{*}{ Gerardin [51] } & $\mathrm{AD}$ vs NC & \multirow{2}{*}{ Hippocampi + SVM } & \multirow{2}{*}{ MRI } & $94.0 \%$ \\
\hline & $\mathrm{MCI}$ vs NC & & & $83.0 \%$ \\
\hline Hackman [52] & MC vs NC & Wavelet Transform + SVM & MRI & $80.44 \%$ \\
\hline \multirow{2}{*}{ Dukart [45] } & \multirow{2}{*}{$\mathrm{AD}$ vs $\mathrm{NC}$} & \multirow{2}{*}{ Meta-Analysis + SVM } & \multirow{2}{*}{ MRI, PET } & $85.7 \%$ \\
\hline & & & & $100.0 \%$ \\
\hline \multirow{3}{*}{ Ortiz[53] } & \multirow{3}{*}{$\mathrm{AD}$ vs NC } & LVQ + SVM & \multirow{3}{*}{ MRI } & $91.0 \%$ \\
\hline & & $\mathrm{PCA}+\mathrm{SVM}$ & & $81.0 \%$ \\
\hline & & $\mathrm{VAF}+\mathrm{SVM}$ & & $71.0 \%$ \\
\hline \multirow{2}{*}{$\operatorname{Nir}[60]$} & $\mathrm{AD}$ vs $\mathrm{NC}$ & \multirow{2}{*}{$\begin{array}{l}\text { Diffusion Weighted method } \\
+ \text { SVM }\end{array}$} & \multirow{2}{*}{ MRI } & $86.2 \%$ \\
\hline & MCI vs NC & & & $82.0 \%$ \\
\hline Schmitter [54] & $\mathrm{AD}$ vs $\mathrm{NC}$ & FreeSurfer + SVM & MRI & NAN \\
\hline
\end{tabular}

of SPECT that resulted in 74\%. In [49] Vemuri et al. utilized SVM as the classification algorithm as well as feature selection technique and attained a sensitivity of $86.0 \%$ whereas $92.0 \%$ specificity in Alzheimer's disease on magnetic resonance images data. In [45] Dukart et al. utilized a meta-analysis centered on support vector machine for diagnosis of Alzheimer's disease and normal control and attain accuracy of $90 \%$, specificity of $87.8 \%$, and sensitivity of $91.8 \%$ on both magnetic resonance images and PET. Magnin et al.[20] utilized Histogram and Support Vector Machine on MRI to distinguish patients with Alzheimer's Disease from other normal subjects and attained accuracy, sensitivity, and specificity of $94.6 \%, 91.5 \%$, and $96.6 \%$ respectively. Fan et al. [50] utilized VBM and non-linear Support Vector Machine on MRI to distinguish patients with SC from other normal subjects and attained an overall accuracy of $90.2 \%$, and with linear SVM attained 88.5\% accuracy. Geradin et al.[51] utilized Hippocampi and Support Vector Machine on MRI scans to distinguish patients with Alzheimer's Disease from other normal subjects and attained accuracy, sensitivity, and specificity of $94.6 \%, 96 \%$, and $92 \%$ respectively. Hackman et al. [52] utilized Wavelet Transform and Support Vector Machine on MRI to classify patients with MC from other normal subjects and attained accuracy, sensitivity, and specificity of $80.44 \%, 87.80 \%$, and $73.08 \%$ respectively. Ortiz et al. [53] utilized VAF and Support Vector Machine on MRI to distinguish patients with MC from other normal subjects and attained accuracy, sensitivity, and specificity of $71.0 \%, 76.0 .80 \%$, and $66.08 \%$ respectively and after VAF.

Schmitter et al.[54] utilized FreeSurfer and Support Vector Machine on MRI to distinguish patients with MC from other normal subjects and attained sensitivity and specificity, $82.80 \%$ and $88.08 \%$ respectively. Horn et al.[55] utilized SVM to distinguished patients with Alzheimer's Disease from other Frontotemporal Dementia (FTD) and attained accuracy, sensitivity, and specificity, $87.0 \%, 88.0 \%$, and $87.08 \%$ respectively. Table II shows the Performance comparison of SVM over the above-mentioned techniques.

\section{Logistic Regression}

Logistic Regression Classification classifies the input samples to their respective classes based on the probabilistic value returned through the logistic sigmoid function. To differentiate Alzheimer's Disease from other sorts of dementia, Logistic Regression Classification is utilized in various Alzheimer's Disease analyses [56]-[58]. Logistic
Regression performs classification for Alzheimer's disease MRI in a similar way to SVM [59]. To design a prediction model for the timely detection and progression of Alzheimer's Diseases, Johnson et al. [61] utilized Logistic Regression Classification (LRC). In the large feature space for finding the optimum features like neuropsychological tests, a genetic algorithm (GA) is utilized [62] [63]. These Optimal features from the Genetic Algorithm are maintained as the inputs of the Logistic Regression Classification (LRC). It appears that the Genetic Algorithm can enhance the detection of Alzheimer's disease. For the detection of different analyses of dementia, a two-level prediction model was submitted by Mazzocco and Hussain [64].

\section{Linear Discriminant Analysis}

Linear Discriminating Analysis (LDA) is thoroughly connected to studies of variance and regression studies which show one dependent variable as a linear combination of other features or dimensions [65]. Linear Discriminating Analysis (LDA) is also called 'Fisher Linear Discriminant " which is the most common size reduction approach [66]. Linear Discriminating Analysis (LDA) develops a linear discriminant function resultant in minimum errors [13], [67], [68].

Zhao et al. [69] suggested an enhanced iterative trace ration (iITR) procedure to resolve the trace ratio linear discriminate analysis (TR-LDA) problematic for dementia analysis and attained improved results as compared to the principal component analysis (PCA), locality preserving projections (LLP), and maximum margin criterion (MMC). In [55] horn et al. utilized the image features reduced by the partial least square (PLS) to LDA for distinguishing AD from FTD and attained an accuracy of $84 \%$, a sensitivity of $83 \%$, and a specificity of $86 \%$ on perfusion SPECT images. Horn et al. [55] utilized PLS and LDA to distinguished patients with Alzheimer's Disease from other FTD by utilizing SPECT images and attained accuracy, sensitivity, and specificity, $80.4 \%, 83.0 \%$, and $86.08 \%$ respectively. Zhao et al.[69] utilized KPCA and TR-LDA to distinguish patients with Alzheimer's Disease from other Normal control and attained an accuracy of $90.01 \%$.

\section{K-means Clustering (Hard Clustering)}

The Clustering (grouping) approach is also known as unsupervised learning [80] [81], as the classification approaches categorized instances in dissimilar groups, but in the clustering approach (unsupervised learning) there is no training dataset to practice [82]. In 
TABLE III. MACHINE LEARNING-BASEd APPROACHES

\begin{tabular}{|c|c|c|c|c|}
\hline Author & Targets & Methods & Imaging Modality & Accuracy \\
\hline J. Akhila et al.[70] & Classification of $\mathrm{AD}$ & Feedforward NN & MRI & $97.5 \%$ \\
\hline C.V Dolph et al. [71] & Classification & SAE DNN & MRI & $56 \%$ \\
\hline Faturrahman et al. [72] & $\mathrm{AD}$ vs $\mathrm{NC}$ & DBN & MRI & $91.7 \%$ \\
\hline H.I.Suk et al. [73] & Features extraction and classification & DESRN & MRI & $90.28 \%$ \\
\hline E.M. Alkabawi et al. $[74,75]$ & Features extraction and classification & $\mathrm{CNN}+\mathrm{LR}$ & MRI & $74.93 \%$ \\
\hline J. Akhila et al.[70] & Features extraction and classification & Feedforward NN & MRI & $97.5 \%$ \\
\hline Cui et al.[76] & $\mathrm{AD}$ diagnosis & RNN & MRI & $89.7 \%$ \\
\hline Wang Yen et al.[77] & $\mathrm{AD}$ vs $\mathrm{NC}$ vs $\mathrm{MCI}$ & $\mathrm{CNN}$ & MRI & $92.06 \%$ \\
\hline Gunawerdena et al.[78] & $\mathrm{AD}$ & $\mathrm{CNN}$ & MRI & $96 \%$ \\
\hline Seixas et al.[25] & $\mathrm{MCI}$ vs $\mathrm{NC}$ & Bayesian Network & MRI & NAN \\
\hline \multirow{3}{*}{ Horn [55] } & \multirow{3}{*}{$\mathrm{AD}$ vs FTD } & PLS + LDA & SPECT & 84.0 \\
\hline & & PLS + K-NN & SPECT & 88.0 \\
\hline & & SVM & SPECT & 87.0 \\
\hline Zhao[69] & Dementia vs NC & KPCA + TR-LDA & - & 90.01 \\
\hline \multirow{2}{*}{ Plant [21] } & $\mathrm{AD}$ vs $\mathrm{NC}$ & Data Mining + SVM & MRI & 90.0 \\
\hline & MCI vs NC & Data Mining + Bayes & MRI & 85.71 \\
\hline Papakostas [79] & $\mathrm{AD}$ vs $\mathrm{NC}$ & VBM V LC-KNN & MRI & 80.0 \\
\hline \multirow{2}{*}{ Liu [26] } & $\mathrm{AD}$ vs NC & \multirow{2}{*}{$\begin{array}{l}\text { Multifold Bayesian } \\
\text { Kernelization }\end{array}$} & MRI, PET & 84.74 \\
\hline & MCI vs MCInc & & MRI, PET & 63.79 \\
\hline
\end{tabular}

medical imaging issues and the detection of Alzheimer's disease, this clustering approach is widely utilized e.g. segmentation of brain tissue, hippocampal division, and entire cerebrum division i.e. segmentation. In [83] authors provided efficiency for various clustering procedures aimed at describing the physiognomies of cerebrum muscles for assessment of Alzheimer's disease in various phases. K-means is an unsupervised learning approach that is mainly utilized when having data without label, which means data having no definite clusters and categories. It is a famous method utilized to assemble in precharacterized digits of $\mathrm{K}$ groups i.e. K-means grouping which is a hard-grouping approach. This procedure aims to find out clusters in the data set. For hippocampal division, cerebrum muscle division, and tumor affected area division (segmentation) K-means is used [79]. To differentiate subjects into pathologic groups Escudero et al. [80] used KM i.e. K-Means by distinguishing EEG temporal measures. Rodrigues et al. [81] isolated cases having AD and normal subjects by utilizing $\mathrm{K}$-means clustering. They observed K-means as the best method for an unsupervised diagnosis of EEG temporal arrangements. To choose the centroid of groups in KM for cerebrum segmentation in Magnetic Resonance Images, a new approach recommended by Liu and Guo [82] is based on shifting average filtering. Papakostas et al. [83] utilized VBM and KNN on MRI to distinguished patients with MC from other normal subjects and attained accuracy, sensitivity, and specificity of $80.44 \%, 80.80 \%$, and $79.08 \%$ respectively. Horn et al. [55] utilized PLS and KNN on SPECT to distinguished patients with Alzheimer's Disease from other FTD and attained accuracy, sensitivity, and specificity of $88.0 \%, 93.0 \%$, and $85.08 \%$ respectively. Table III shows the performance comparison of distinct machine learning-based existing techniques.

\section{Deep Learning-Based Approaches}

\section{Transfer Learning}

Transfer Learning (TL) is a well-known Machine Learning approach in which a model that is trained can be reutilized on another related task. Because of not having enough data, numerous studies had anticipated methods to upsurge openly existing data, which are summarized in Table IV. K. Aderghal et al. in [84] proposed transfer learning from transferring the info from sMRI data to DTI images. They trained the model on SMRI with extensive distinct augmentation techniques and then transferred the info to the DTI dataset by utilizing the ADNI dataset repository for Normal subject classification, AD, and MCI. The first subject of images with just structural magnetic resonance images sMRI was chosen from the ADNI-1 repository and the second subject of images was taken from ADNI GO and ADNI. This second subject consisted of structural magnetic resonance images along with the diffusion tensor images. The second subset of images included the group of subjects with both sMRI and the DTI modalities. In this study, the hippocampus region was in focus which is a regular functional structure of the cerebrum containing two sections. To acquire just region of interest (RoI) for given $\mathrm{AD}$ individuals, they measured the average of two sections when floating the right region of the hippocampus to the left region. Data augmentation was used as a preprocessing step and all the experiments were done by utilizing the Café Deep learning framework. They attained classification accuracies of $92.5 \%$ for $\mathrm{AD}$ vs NC, $85.0 \%$ for $\mathrm{AD}$ vs $\mathrm{MCI}$, and $80.0 \%$ for Mild Cognitive Impairment vs NC.

In [85], Afzal et al. proposed a new approach by utilizing image augmentation-based techniques to classify the AD utilizing the OASIS dataset. They performed all the experiments using transfer learning and attained $98.2 \%$ performance accuracy. In [86] N.M et al. proposed a transfer learning approach for the prediction of the binary class Alzheimer's Disease and Maqsood et al. [87] proposed a transfer learning approach by utilizing pre-trained AlexNet for multiclass classification of Alzheimer's Disease.

T. D. Phong et al. [88] established the efficacy of utilizing pretrained models as a starting point for other networks. The other two models proposed in research, i.e. GoogNet and ResNet, are reinforced by python's TensorFlow library2 and are pre-trained with ImageNet, having great expertise to distinguish numerous types of real images. Later, those models utilized in this research were only trained for the fully connected layers of the network. S. Wang, et al. [89] utilize the TL approaches and Augmentation to resolve the inadequate samples of the data to identify Mild Cognitive Impairment on Magnetic Resonance Images. They utilize OASIS2 and their performance accuracy was $90.6 \%$ for MCI vs Normal Control. The Diffusion Tensor Images maps are frequently understood as upright modularity for $\mathrm{AD}$ identification. So M. A. Nowrangi et al. in [90] have compared the NC, AD, and MCI. The outputs showed MD is a good indicator. B. Cheng et al. [91] use MCI vs structural Mild Cognitive Impairment as the Supplementary domain 
TABLE IV. Transfer LeARning-BASEd Approaches

\begin{tabular}{lllll}
\hline Author & Method & Dataset & Images & Accuracy \\
\hline Afzal et al. [85] & Transfer Learning & OASIS & MRI & $98.41 \%$ \\
\hline Muazzam et al. [87] & Transfer Learning & OASIS & MRI & $92.85 \%$ \\
\hline N. M et al [86] & Transfer Learning & OASIS & MRI & $99.4 \%$ \\
\hline & Transfer Learning & & $92.5 \%$ AD vs NC \\
\cline { 3 - 4 } K. Aderghal et al. [84] & sMRI to DTI & ADNI & MRI & $85.0 \%$ AD vs MCI \\
\cline { 3 - 5 } & Transfer Learning & 115 different hospitals & CT scans & NAN \\
\hline T. D. Phong et al. [88] & Transfer Learning & OASIS & $90.0 \%$ \\
\hline S. Wang, et al. [89] & Multi-Domain Transfer Learning & ADNI & MRI \\
\hline B. Cheng et al. [91] & ImageNet Transfer Learning & ADNI & MRI & $83.7 \%$ \\
\hline T. Glozman et al. [92] & FA and MD & ADNI & MRI & $83 \%$ \\
\hline M. Dyrba et al. [93] & AD classification & ADNI & MRI & $84 \%$ with adaptation \\
\hline Li et al. [94] & & & \\
\hline
\end{tabular}

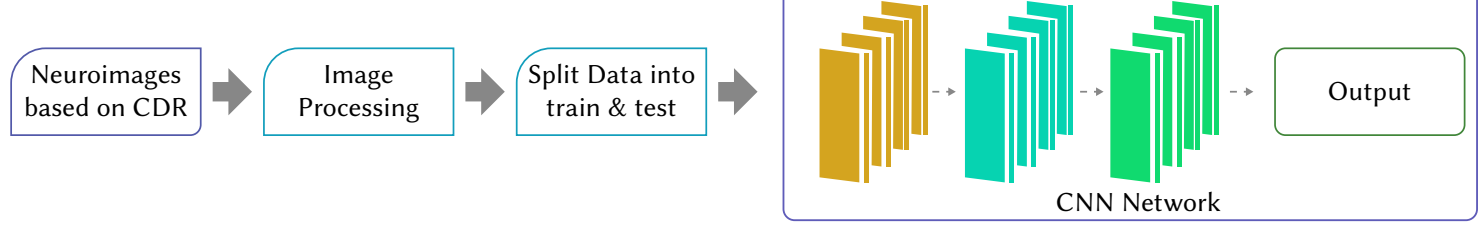

Fig. 5. The fundamental framework for the deep learning approach for the classification of $A D$.

for classification of Normal Control vs Alzheimer's Disease The main idea of our multi-domain transfer learning-based method is to exploit the multi-auxiliary domain data to enhance the classification of the targeted domain. They then linked the multi_domain and the target domain to enhance the selection and classification of features phase. The accuracy for AD vs NC was $94 \%$ and for MCI vs NC, they attained $82.1 \%$ classification accuracy. T. Glozman et al. in [92] utilized the pretrained ImageNet as the source domain, by utilizing the full brain with joint classification. Only initial layers were transferred. They attained an accuracy of $83.5 \%$ for AD vs NC. M. Dyrba et al. [93] utilized FA and MD with the SVM, utilizing the info gain measure approach for $\mathrm{AD}$ classification. For FA, the accuracy was $80 \%$ whereas the accuracy was $83 \%$ in the case of MD. Li et al. [94] also proposed the approach for the classification of this disease for small datasets on a knowledge transfer perspective and attained accuracies of $49.0 \%$ with Tongi, $61.5 \%$ using naïve combination, $55.3 \%$ using insane weighting, and $84 \%$ using with adaptation. Table IV shows the Performance comparison of Transfer Learning for deep learning-based existing techniques. LDA was used for $\mathrm{AD}$ classification using different features. The results are evaluated on ADNI and 63.7\% accuracy was achieved. Another study combined the features from CSF, GM, and WM and performed classification [95]. These features were used for AD stage classification over the ADNI dataset with $79.8 \%$ accuracy [96]. D. Chitradevi et al. [97] proposed a method using multiple sub-regions of the cerebral and these subregions were WM, GM, and hippocampus. Different classification methods including PSA and gray wolf optimization were used for AD classification with $98 \%$ accuracy. Hao et al. [98] performed AD stage detection using thresholding with 95\% accuracy. Chihun Park et al. [99] worked on a similar algorithm for $\mathrm{AD}$ stage classification and achieved $82.3 \%$ accuracy. Arifa et al. [100] proposed a system using $\mathrm{CNN}$ and hybrid features for $\mathrm{AD}$ and achieved very good results.

\section{Convolutional Neural Network}

In [76], researchers present an MLP combinational framework, multilayer perception, and RNN identification of this disease by using MRI. Initially, MLP is used and then authors use the 2-level RNN formed on the MLP. They achieved an accuracy of $89.7 \%$ for the AD classification. For all these experiments, they use ADNI data sets.

Wang Yan et al. [77] use three-class subjects with balanced sample sizes and achieved greater precision by combining multimode magnetic resonance with the CNN core. They achieved $92.06 \%$ accuracy for this experiment. Gunawardena et al. [101] used a total of 1615 scans and reached $84.4 \%$ accuracy by utilizing the vector carrier and then proposed the CNN approach and attained an accuracy of $96 \%$. This section discussed the application of pre-processing techniques over brain images utilized in the existing literature. Each of the techniques considering $\mathrm{AD}$ detection is discussed and their representative articles are also briefly overviewed. The section in its second half also discussed the utilized machine learning algorithms for efficient and accurate segmentation and classification results. All the techniques were comparatively analyzed, and their performance was represented in tabular form. A generic CNN framework is presented in Fig. 5.

\section{Datasets and Softwares}

Diverse freely online-available datasets and programming bundles are accessible to help. Mind picture investigation bundles, for example, FreeSurfer (surfer.nmr.mgh), FSL (https://fsl.fmrib.ox.ac. $\mathrm{uk} /$ ), MIPAV(mipav.cit.nih.gov), and SPM, which give integral assets to various robotized pre-preparing systems [102]. Additionally, programming bundles, for example, MATLAB (mathworks.com), Keras (keras.io), Tensorflow (tensorflow.org), Theano (deeplearning.net/ programming/ theano), Caffe (caffe.berkeleyvision.org), and Torch (torch.ch) are utilized to execute profound frameworks [103]-[105] Also, freely online-available, for example, ADNI [106], AIBL [107], and OASIS [108] are exceptionally useful too. These datasets make freely functional attractive reverberation images of the brain. Fig. 6 represents the overall utilization of the tools for the given problem.

\section{A. ADNI Datasets}

The data of Alzheimer's disease Neuroimaging Initiative (ADNI), a database available at (adni.loni.ucla.edu), were used throughout in every Alzheimer's detection-related study. The dataset of ADNI was launched in 2003 by the National Institute on Aging (NIA), the Food and Drug Administration (FDA), the National Institute of Biomedical Imaging and Bioengineering (NIBIB) private non-profits, and pharmaceutical companies of the private sector. The main role of the ADNI repository has been to analyze its images whether serial 


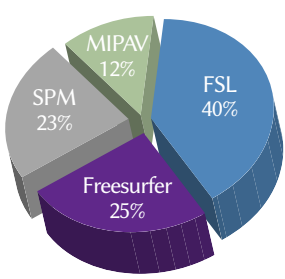

a

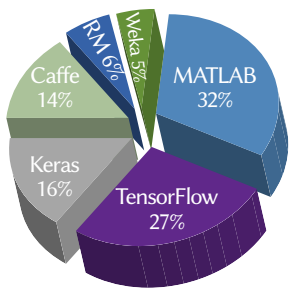

b
Fig. 6. (a) Pie Diagram showing the utilization ratio of different software for preprocessing (b) Pie Diagram showing the utilization ratio of different software for classification purposes.

magnetic resonance imaging (MRI), positron emission tomography (PET), other clinical and neuropsychological, and biological markers.

\section{B. OASIS Datasets}

The dataset of the Open Access Series of Imaging Studies (OASIS) is an open-source repository for the scientific community for Alzheimer's disease classification. The basic reason for the compilation of this dataset is to facilitate future discoveries in neurodegenerative disease, which is based on a multi-model-based dataset. Formally released data for OASIS-Cross-sectional and OASIS-Longitudinal have been used for hypothesis-driven data analyses, development of neuroanatomical atlases, and development of segmentation algorithms. OASIS is a neuroimaging-based data of longitudinal dimension, clinical dimension data, cognitive-based data, and biomarker dataset. This dataset is formed representing progressive stages of the disease from normal to mild, moderate to severe stages of the dataset. These stages can be distinguished in their clinical dementia rating (CDR) representing the overall classes. The OASIS dataset is hosted on which provides open access to the substantial database of processed MRI images and neuroimaging for the community. This dataset is a broad demographic, genetic spectrum, and cognitive-based dataset. This can be used for neuroimaging-based clinical and cognitive research purposes. This is a multi-staged dataset range from normal aging to cognitive decline. A broad horizon of studies in this domain is based on the OASIS dataset. The dataset can be accessed via https://www.oasis-brains.org/.

\section{Other Neuroimaging Dataset}

Apart from open-access datasets, the remaining studies dataset was collected from diverse open source scanners based on over the diverse sources across the spectrum for diverse Alzheimer's disease detection and other health infections [109]-[111]. The details of each respective study dataset are comprised of multiple $\mathrm{T}$ weighted magnetic resonance imaging (MRI), positron emission tomography (PET) scans.

Each study uses and selects a dataset according to the need and requirement of the algorithm to fully scale the problem statement under consideration. In this study two main data repositories, ADNI, and OASIS were used broadly as they are available publicly and can be used with ease. OASIS and ADNI dataset images are collected and processed with a more domain-centric approach. As the OASIS and ADNI datasets have millions of MRIs and PET images with dedicated domains, these datasets are used widely for experimental studies. This section discusses the datasets utilized in our discussed techniques in existing literature regarding the detection and classification of $\mathrm{AD}$. The section also provides links to the data repositories for access to publicly available datasets.

\section{Discussion And Future Direction}

In this section, we have discussed the most important features for the identification of $\mathrm{AD}$. Alzheimer's disease is neurodegenerative dementia and has an impact on the fitness of an individual. It affects the parts of the brain related to memory and sensing such as the hippocampus, amygdala, parietal lobe, temporal lobe, etc. This paper efficiently presents procedures for improving $\mathrm{AD}$ diagnosis by using various approaches to image processing, machine learning, and deep learning. Firstly, classifying the early Mild-Cognitive-Impairments individuals from normal controls and its conversion to Alzheimer's are most significant. This timely identification at the initial stage of this disease would propose so many possible advantages to the individual. Over the last decade, numerous contributions have been done by utilizing different machine learning and deep learning approaches, but still, early detection remains an issue for $\mathrm{AD}$ diagnosis, instead of considering the whole brain part, some studies focus only on the Region of Interest part. This RoI-based has low feature dimension and can be easily interpreted. The performance of $\mathrm{AD}$ diagnosis is greatly based on the quality of the neuroimages. Neuroimaging modalities including MRI, fMRI, and PET are essential to Alzheimer's detection. This neuro-imaging identification for Alzheimer's is being a complex procedure involving countless influences that rely on these image modalities. Various other aspects, for example, age in years, gender, and education level are also useful. The most influential transfer learning models are mainly AlexNet, LeNet, and GoogleNet. There are a lot of open-source libraries that can be deployed to explore these models, as these are fast and efficient. Currently, neural network transfer learning methods are extensively used but the unavailability of enough data samples is a problem for the $\mathrm{AD}$ classification.

This approach is the most prominent and emerging technique in the machine learning field. The Convolutional Neural Network is another most prominent and state-of-the-art technology in $\mathrm{AD}$ diagnosis. These CNN approaches are widely used in many other computervision tasks including classification. The Convolutional Neural Network-based approaches are the most efficient and significant method for large scale issues including classification of more than 1000 classes, without handcrafted features, CNN works automatically to classify the distinct classes but the main issue in deep learning is that it requires huge training data to train the network, sometimes it is difficult to tune the $\mathrm{CNN}$ because of the hyperparameters. If the training data has not enough data samples then the overfitting issue may occur. The number of Alzheimer's individuals, as well as MCI, could be very limited in each dataset, which is inadequate for testing a deep learning framework. This circumstance is more terrible for multimodality studies. Therefore, some research combines the datasets to avoid this class imbalance issue. Another way to resolve this class imbalance issue is to apply distinct augmentation approaches. Data augmentation is an efficient approach that increases the number of samples in the training set without gathering new data.

The future directions for $\mathrm{AD}$ classification include more localized pattern recognition to identify the changes in the brain. For this purpose, the researchers can divide the images into multiple equal parts for feature extraction and $\mathrm{AD}$ classification. The $\mathrm{AD}$ classification can be further improved by combining multiple modalities and by using reinforcement learning.

\section{ACKNOWLEDGMENT}

"This work was supported by the Soonchunhyang University Research Fund."

\section{REFERENCES}

[1] M. A. Binnewijzend, M. M. Schoonheim, E. Sanz-Arigita, A. M. Wink, W. M. van der Flier, N. Tolboom, et al., "Resting-state fMRI changes in Alzheimer's disease and mild cognitive impairment," Neurobiology 
of aging, vol. 33, pp. 2018-2028, 2012. https://doi.org/10.1016/j. neurobiolaging.2011.07.003

[2] H. Braak and E. Braak, "Neuropathological stageing of Alzheimer-related changes," Acta neuropathologica, vol. 82, pp. 239-259, 1991. https://doi. org/10.1007/BF00308809

[3] E. E. Bron, M. Smits, W. M. Van Der Flier, H. Vrenken, F. Barkhof, P. Scheltens, et al., "Standardized evaluation of algorithms for computeraided diagnosis of dementia based on structural MRI: the CADDementia challenge," NeuroImage, vol. 111, pp. 562-579, 2015 https://doi. org/10.1016/j.neuroimage.2015.01.048

[4] V. Camus, P. Payoux, L. Barré, B. Desgranges, T. Voisin, C. Tauber, et al., "Using PET with 18 F-AV-45 (florbetapir) to quantify brain amyloid load in a clinical environment," European journal of nuclear medicine and molecular imaging, vol. 39, pp. 621-631, 2012. https://doi.org/10.1007/ s00259-011-2021-8

[5] R. C. Petersen, G. E. Smith, S. C. Waring, R. J. Ivnik, E. G. Tangalos, and E. Kokmen, "Mild cognitive impairment: clinical characterization and outcome," Archives of neurology, vol. 56, pp. 303-308, 1999. doi:10.1001/ archneur.56.3.303

[6] A. s. Disease and R. D. Association, 2017 Alzheimer's Disease Facts and Figures: Includes a Special Report on the Next Frontier of Alzheimer's Research: Alzheimer's Association, 2017. https://doi.org/10.1016/j. jalz.2017.02.006

[7] J. P. Lerch, J. Pruessner, A. P. Zijdenbos, D. L. Collins, S. J. Teipel, H. Hampel, et al., "Automated cortical thickness measurements from MRI can accurately separate Alzheimer's patients from normal elderly controls," Neurobiology of aging, vol. 29, pp. 23-30, 2008. https://doi.org/10.1016/j.neurobiolaging.2006.09.013

[8] S. Klöppel, C. M. Stonnington, C. Chu, B. Draganski, R. I. Scahill, J. D. Rohrer, et al., "Automatic classification of MR scans in Alzheimer's disease," Brain, vol. 131, pp. 681-689, 2008. https://doi.org/10.1093/brain/ awm319

[9] R. Brookmeyer, E. Johnson, K. Ziegler-Graham, and H. M. Arrighi, "Forecasting the global burden of Alzheimer's disease," Alzheimer's \& dementia, vol. 3, pp. 186-191, 2007. https://doi.org/10.1016/j. jalz.2007.04.381

[10] R. L. Buckner, "Memory and executive function in aging and AD: multiple factors that cause decline and reserve factors that compensate," Neuron, vol. 44, pp. 195-208, 2004. https://doi.org/10.1016/j.neuron.2004.09.006

[11] G. F. Busatto, G. E. Garrido, O. P. Almeida, C. C. Castro, C. H. Camargo, C. G. Cid, et al., "A voxel-based morphometry study of temporal lobe gray matter reductions in Alzheimer's disease," Neurobiology of aging, vol. 24, pp. 221-231, 2003. https://doi.org/10.1016/S0197-4580(02)00084-2

[12] C. Cabral, P. M. Morgado, D. C. Costa, M. Silveira, and A. s. D. N. Initiative, "Predicting conversion from MCI to AD with FDG-PET brain images at different prodromal stages," Computers in biology and medicine, vol. 58, pp. 101-109, 2015. https://doi.org/10.1016/j.compbiomed.2015.01.003

[13] H. Wang, S. Yan, D. Xu, X. Tang, and T. Huang, "Trace ratio vs. ratio trace for dimensionality reduction," in Computer Vision and Pattern Recognition, 2007. CVPR'07. IEEE Conference on, 2007, pp. 1-8. DOI: $10.1109 /$ CVPR.2007.382983

[14] M. D. Fox and M. E. Raichle, "Spontaneous fluctuations in brain activity observed with functional magnetic resonance imaging," Nature reviews neuroscience, vol. 8, p. 700, 2007. https://doi.org/10.1038/nrn2201

[15] M. D. Greicius, B. Krasnow, A. L. Reiss, and V. Menon, "Functional connectivity in the resting brain: a network analysis of the default mode hypothesis," Proceedings of the National Academy of Sciences, vol. 100, pp. 253-258, 2003. https://doi.org/10.1073/pnas.0135058100

[16] P. T. Fox, A. R. Laird, and J. L. Lancaster, "Coordinate-based voxel-wise meta-analysis: Dividends of spatial normalization. Report of a virtual workshop," Human brain mapping, vol. 25, pp. 1-5, 2005. DOI: 10.1002/ hbm.20139

[17] Y. He, L. Wang, Y. Zang, L. Tian, X. Zhang, K. Li, et al., "Regional coherence changes in the early stages of Alzheimer's disease: a combined structural and resting-state functional MRI study," Neuroimage, vol. 35, pp. 488-500, 2007. https://doi.org/10.1016/j.neuroimage.2006.11.042

[18] S. Teipel, A. Drzezga, M. J. Grothe, H. Barthel, G. Chételat, N. Schuff, et al., "Multimodal imaging in Alzheimer's disease: validity and usefulness for early detection," The Lancet Neurology, vol. 14, pp. 1037-1053, 2015. https://doi.org/10.1016/S1474-4422(15)00093-9
[19] Y. Chen, D. Wolk, J. Reddin, M. Korczykowski, P. Martinez, E. Musiek, et al., "Voxel-level comparison of arterial spin-labeled perfusion MRI and FDG-PET in Alzheimer disease," Neurology, p. WNL. 0b013e31823a0ef7, 2011. DOI: https://doi.org/10.1212/WNL.0b013e31823a0ef7

[20] B. Magnin, L. Mesrob, S. Kinkingnéhun, M. Pélégrini-Issac, O. Colliot, M. Sarazin, et al., "Support vector machine-based classification of Alzheimer's disease from whole-brain anatomical MRI," Neuroradiology, vol. 51, pp. 73-83, 2009. DOIhttps://doi.org/10.1007/s00234-008-0463-x

[21] C. Plant, S. J. Teipel, A. Oswald, C. Böhm, T. Meindl, J. Mourao-Miranda, et al., "Automated detection of brain atrophy patterns based on MRI for the prediction of Alzheimer's disease," Neuroimage, vol. 50, pp. 162-174, 2010. https://doi.org/10.1016/j.neuroimage.2009.11.046

[22] J. Friedrich, R. Urbanczik, and W. Senn, "Code-specific learning rules improve action selection by populations of spiking neurons," International journal of neural systems, vol. 24, p. 1450002, 2014. https:// doi.org/10.1142/S0129065714500026

[23] G. Lee, M. Kwon, S. Kavuri, and M. Lee, "Action-perception cycle learning for incremental emotion recognition in a movie clip using 3D fuzzy GIST based on visual and EEG signals," Integrated Computer-Aided Engineering, vol. 21, pp. 295-310, 2014. DOI: 10.3233/ICA-140464

[24] U. S. Shanthamallu, A. Spanias, C. Tepedelenlioglu, and M. Stanley, "A brief survey of machine learning methods and their sensor and IoT applications," in Information, Intelligence, Systems \& Applications (IISA), 2017 8th International Conference on, 2017, pp. 1-8. DOI: 10.1109/ IISA.2017.8316459

[25] F. L. Seixas, B. Zadrozny, J. Laks, A. Conci, and D. C. M. Saade, "A Bayesian network decision model for supporting the diagnosis of dementia, Alzheimer' s disease and mild cognitive impairment," Computers in biology and medicine, vol. 51, pp. 140-158, 2014. https://doi.org/10.1016/j. compbiomed.2014.04.010

[26] S. Liu, Y. Song, W. Cai, S. Pujol, R. Kikinis, X. Wang, et al., "Multifold Bayesian kernelization in Alzheimer's diagnosis," in International Conference on Medical Image Computing and Computer-Assisted Intervention, 2013, pp. 303-310. DOIhttps://doi.org/10.1007/978-3-64240763-5_38

[27] M. Catá Villá, "Feature selection methods for predicting pre-clinical stage in Alzheirmer's Disease," Universitat Politècnica de Catalunya, 2014.

[28] M. López, J. Ramírez, J. Górriz, D. Salas-Gonzalez, I. Alvarez, F. Segovia, et al., "Automatic tool for Alzheimer's disease diagnosis using PCA and Bayesian classification rules," Electronics Letters, vol. 45, pp. 389-391, 2009. DOI: 10.1049/el.2009.0176,

[29] F. Pereira, T. Mitchell, and M. Botvinick, "Machine learning classifiers and fMRI: a tutorial overview," Neuroimage, vol. 45, pp. S199-S209, 2009. https://doi.org/10.1016/j.neuroimage.2008.11.007

[30] V. Vapnik, The nature of statistical learning theory: Springer science \& business media, 2013.

[31] G. Mirzaei, A. Adeli, and H. Adeli, "Imaging and machine learning techniques for diagnosis of Alzheimer's disease," Reviews in the Neurosciences, vol. 27, pp. 857-870, 2016. DOI: https://doi.org/10.1515/ revneuro-2016-0029

[32] H. Furuta, K. Maeda, and E. Watanabe, "Application of genetic algorithm to aesthetic design of bridge structures," Computer-Aided Civil and Infrastructure Engineering, vol. 10, pp. 415-421, 1995. https://doi. org/10.1111/j.1467-8667.1995.tb00301.x

[33] J. S. Chou and A. D. Pham, "Smart artificial firefly colony algorithm-based support vector regression for enhanced forecasting in civil engineering," Computer-Aided Civil and Infrastructure Engineering, vol. 30, pp. 715732, 2015.

[34] H. Adeli, Advances in design optimization: CRC press, 1994.

[35] O. Chapelle, V. Sindhwani, and S. S. Keerthi, "Optimization techniques for semi-supervised support vector machines," Journal of Machine Learning Research, vol. 9, pp. 203-233, 2008.

[36] V.Vural and J. G. Dy, "A hierarchical method for multi-class support vector machines," in Proceedings of the twenty-first international conference on Machine learning, 2004, p. 105. https://doi.org/10.1145/1015330.1015427

[37] J. D. Haynes and G. Rees, "Neuroimaging: decoding mental states from brain activity in humans," Nature Reviews Neuroscience, vol. 7, p. 523, 2006. DOIhttps://doi.org/10.1038/nrn1931

[38] S. Afzal, M. Javed, M. Maqsood, F. Aadil, S. Rho, and I. Mehmood, "A Segmentation-Less Efficient Alzheimer Detection Approach Using 
Hybrid Image Features," in Handbook of Multimedia Information Security: Techniques and Applications, ed: Springer, 2019, pp. 421-429. DOI: https://doi.org/10.1007/978-3-030-15887-3_20

[39] S. Bauer, L.-P. Nolte, and M. Reyes, "Fully automatic segmentation of brain tumor images using support vector machine classification in combination with hierarchical conditional random field regularization," in International Conference on Medical Image Computing and ComputerAssisted Intervention, 2011, pp. 354-361. https://doi.org/10.1007/978-3642-23626-6_44

[40] D. G. Lowe, "Distinctive image features from scale-invariant keypoints," International journal of computer vision, vol. 60, pp. 91-110, 2004. https:// doi.org/10.1023/B:VISI.0000029664.99615.94

[41] G. Orru, W. Pettersson-Yeo, A. F. Marquand, G. Sartori, and A. Mechelli, "Using support vector machine to identify imaging biomarkers of neurological and psychiatric disease: a critical review," Neuroscience \& Biobehavioral Reviews, vol. 36, pp. 1140-1152, 2012. https://doi. org/10.1016/j.neubiorev.2012.01.004

[42] T. Smith-Vikos and F. J. Slack, "MicroRNAs circulate around Alzheimer's disease," Genome biology, vol. 14, p. 125, 2013. https://doi.org/10.1186/ gb-2013-14-7-125

[43] C. Laske, T. Leyhe, E. Stransky, N. Hoffmann, A. J. Fallgatter, and J. Dietzsch, "Identification of a blood-based biomarker panel for classification of Alzheimer's disease," International Journal of Neuropsychopharmacology, vol. 14, pp. 1147-1155, 2011. https://doi. org/10.1017/S1461145711000459

[44] M. Lopez, J. Ramirez, J. Gorriz, D. Salas-Gonzalez, I. Á. Lvarez, F. Segovia, et al., "Neurological image classification for the Alzheimer's Disease diagnosis using Kernel PCA and Support Vector Machines," in Nuclear Science Symposium Conference Record (NSS/MIC), 2009 IEEE, 2009, pp. 2486-2489. 10.1109/NSSMIC.2009.5402069

[45] J. Dukart, K. Mueller, H. Barthel, A. Villringer, O. Sabri, M. L. Schroeter, et al., "Meta-analysis based SVM classification enables accurate detection of Alzheimer's disease across different clinical centers using FDG-PET and MRI," Psychiatry Research: Neuroimaging, vol. 212, pp. 230-236, 2013. https://doi.org/10.1016/j.pscychresns.2012.04.007

[46] Y. Zhang, S. Wang, and Z. Dong, "Classification of Alzheimer disease based on structural magnetic resonance imaging by kernel support vector machine decision tree," Progress In Electromagnetics Research, vol. 144, pp. 171-184, 2014.

[47] P. Padilla, M. López, J. M. Górriz, J. Ramirez, D. Salas-Gonzalez, and I. Álvarez, "NMF-SVM based CAD tool applied to functional brain images for the diagnosis of Alzheimer's disease," IEEE Transactions on medical imaging, vol. 31, pp. 207-216, 2012. DOI: 10.1109/TMI.2011.2167628

[48] L. K. Ferreira, J. M. Rondina, R. Kubo, C. R. Ono, C. C. Leite, J. Smid, et al., "Support vector machine-based classification of neuroimages in Alzheimer's disease: direct comparison of FDG-PET, rCBF-SPECT and MRI data acquired from the same individuals," Revista Brasileira de Psiquiatria, pp. 0-0, 2017. https://doi.org/10.1590/1516-4446-2016-2083

[49] P. Vemuri, J. L. Gunter, M. L. Senjem, J. L. Whitwell, K. Kantarci, D. S. Knopman, et al., "Alzheimer's disease diagnosis in individual subjects using structural MR images: validation studies," Neuroimage, vol. 39, pp. 1186-1197, 2008. https://doi.org/10.1016/j.neuroimage.2007.09.073

[50] Y. Fan, D. Shen, R. C. Gur, R. E. Gur, and C. Davatzikos, "COMPARE: classification of morphological patterns using adaptive regional elements," IEEE transactions on medical imaging, vol. 26, pp. 93-105, 2007. DOI: $10.1109 /$ TMI.2006.886812

[51] E. Gerardin, G. Chételat, M. Chupin, R. Cuingnet, B. Desgranges, H.S. Kim, et al., "Multidimensional classification of hippocampal shape features discriminates Alzheimer's disease and mild cognitive impairment from normal aging," Neuroimage, vol. 47, pp. 1476-1486, 2009. https://doi. org/10.1016/j.neuroimage.2009.05.036

[52] K. Hackmack, F. Paul, M. Weygandt, C. Allefeld, J.-D. Haynes, and A. s. D. N. Initiative, "Multi-scale classification of disease using structural MRI and wavelet transform," Neuroimage, vol. 62, pp. 48-58, 2012. https://doi. org/10.1016/j.neuroimage.2012.05.022

[53] A. Ortiz, J. M. Górriz, J. Ramírez, F. J. Martínez-Murcia, and A. s. D. N. Initiative, "LVQ-SVM based CAD tool applied to structural MRI for the diagnosis of the Alzheimer's disease," Pattern Recognition Letters, vol. 34, pp. 1725-1733, 2013. https://doi.org/10.1016/j.patrec.2013.04.014

[54] D. Schmitter, A. Roche, B. Maréchal, D. Ribes, A. Abdulkadir, M. BachCuadra, et al., "An evaluation of volume-based morphometry for prediction of mild cognitive impairment and Alzheimer's disease," NeuroImage: Clinical, vol. 7, pp. 7-17, 2015. https://doi.org/10.1016/j. nicl.2014.11.001

[55] J.-F. Horn, M.-O. Habert, A. Kas, Z. Malek, P. Maksud, L. Lacomblez, et al., "Differential automatic diagnosis between Alzheimer's disease and frontotemporal dementia based on perfusion SPECT images," Artificial intelligence in medicine, vol. 47, pp. 147-158, 2009. https://doi. org/10.1016/j.artmed.2009.05.001

[56] A. Rao, Y. Lee, A. Gass, and A. Monsch, "Classification of Alzheimer's Disease from structural MRI using sparse logistic regression with optional spatial regularization," in Engineering in Medicine and Biology Society, EMBC, 2011 Annual International Conference of the IEEE, 2011, pp. 4499-4502. DOI: 10.1109/IEMBS.2011.6091115

[57] S. Kato, A. Homma, T. Sakuma, and M. Nakamura, "Detection of mild Alzheimer's disease and mild cognitive impairment from elderly speech: Binary discrimination using logistic regression," in Engineering in Medicine and Biology Society (EMBC), 2015 37th Annual International Conference of the IEEE, 2015, pp. 5569-5572. DOI: 10.1109/ EMBC.2015.7319654

[58] X. Zhang, B. Hu, X. Ma, and L. Xu, "Resting-state whole-brain functional connectivity networks for mci classification using 12-regularized logistic regression," IEEE transactions on nanobioscience, vol. 14, pp. 237-247, 2015. DOI: $10.1109 /$ TNB.2015.2403274

[59] R. Casanova, F.-C. Hsu, M. A. Espeland, and A. s. D. N. Initiative, "Classification of structural MRI images in Alzheimer's disease from the perspective of ill-posed problems," PloS one, vol. 7, p. e44877, 2012. https://doi.org/10.1371/journal.pone.0044877

[60] T. M. Nir, J. E. Villalon-Reina, G. Prasad, N. Jahanshad, S. H. Joshi, A. W. Toga, et al., "Diffusion weighted imaging-based maximum density path analysis and classification of Alzheimer's disease," Neurobiology of aging, vol. 36, pp. S132-S140, 2015. https://doi.org/10.1016/j. neurobiolaging.2014.05.037

[61] P. Johnson, L. Vandewater, W. Wilson, P. Maruff, G. Savage, P. Graham, et al., "Genetic algorithm with logistic regression for prediction of progression to Alzheimer's disease," BMC bioinformatics, vol. 15, p. S11, 2014. https://doi.org/10.1186/1471-2105-15-S16-S11

[62] H. G. Lee, C. Y. Yi, D. E. Lee, and D. Arditi, "An advanced stochastic time-cost tradeoff analysis based on a CPM-guided genetic algorithm," Computer-Aided Civil and Infrastructure Engineering, vol. 30, pp. 824842, 2015. https://doi.org/10.1111/mice. 12148

[63] M. Martínez-Ballesteros, J. Bacardit, A. Troncoso, and J. C. Riquelme, "Enhancing the scalability of a genetic algorithm to discover quantitative association rules in large-scale datasets," Integrated Computer-Aided Engineering, vol. 22, pp. 21-39, 2015. DOI: 10.3233/ICA-180580

[64] T. Mazzocco and A. Hussain, "Novel logistic regression models to aid the diagnosis of dementia," Expert Systems with Applications, vol. 39, pp. 3356-3361, 2012. https://doi.org/10.1016/j.eswa.2011.09.023

[65] R. A. Fisher, "The use of multiple measurements in taxonomic problems," Annals of eugenics, vol. 7, pp. 179-188, 1936.

[66] K. Fukunaga, "Introduction to statistical pattern classification," ed: Academic Press USA:, 1990. https://doi.org/10.1117/12.737157

[67] F. Nie, S. Xiang, and C. Zhang, "Neighborhood MinMax Projections," in IJCAI, 2007, pp. 993-998.

[68] S. Xiang, F. Nie, and C. Zhang, "Learning a Mahalanobis distance metric for data clustering and classification," Pattern Recognition, vol. 41, pp. 3600-3612, 2008. https://doi.org/10.1016/j.patcog.2008.05.018

[69] M. Zhao, R. H. Chan, P. Tang, T. W. Chow, and S. W. Wong, "Trace ratio linear discriminant analysis for medical diagnosis: a case study of dementia," IEEE signal processing letters, vol. 20, p. 431, 2013. DOI: 10.1109/LSP.2013.2250281

[70] J. Akhila, C. Markose, and R. Aneesh, "Feature extraction and classification of Dementia with neural network," in 2017 International Conference on Intelligent Computing, Instrumentation and Control Technologies (ICICICT), 2017, pp. 1446-1450. DOI: 10.1109/RBME.2018.2886237

[71] C. V. Dolph, M. Alam, Z. Shboul, M. D. Samad, and K. M. Iftekharuddin, "Deep learning of texture and structural features for multiclass Alzheimer's disease classification," in 2017 International Joint Conference on Neural Networks (IJCNN), 2017, pp. 2259-2266. DOI: 10.1109/ IJCNN.2017.7966129

[72] M. Faturrahman, I. Wasito, N. Hanifah, and R. Mufidah, "Structural 
MRI classification for Alzheimer's disease detection using deep belief network," in 2017 11th International Conference on Information \& Communication Technology and System (ICTS), 2017, pp. 37-42. DOI: 10.1109/ICTS.2017.8265643

[73] H.-I. Suk, S.-W. Lee, D. Shen, and A. s. D. N. Initiative, "Deep ensemble learning of sparse regression models for brain disease diagnosis," Medical image analysis, vol. 37, pp. 101-113, 2017. https://doi.org/10.1016/j. media.2017.01.008

[74] E. M. Alkabawi, A. R. Hilal, and O. A. Basir, "Feature abstraction for early detection of multi-type of dementia with sparse auto-encoder," in 2017 IEEE International Conference on Systems, Man, and Cybernetics (SMC), 2017, pp. 3471-3476. DOI: 10.1109/SMC.2017.8123168

[75] E. M. Alkabawi, A. R. Hilal, and O. A. Basir, "Computer-aided classification of multi-types of dementia via convolutional neural networks," in 2017 IEEE International Symposium on Medical Measurements and Applications (MeMeA), 2017, pp. 45-50. DOI: 10.1109/ MeMeA.2017.7985847

[76] R. Cui, M. Liu, and G. Li, "Longitudinal analysis for Alzheimer's disease diagnosis using RNN," in Biomedical Imaging (ISBI 2018), 2018 IEEE 15th International Symposium on, 2018, pp. 1398-1401. https://doi. org/10.1016/j.media.2020.101694

[77] Y. Wang, Y. Yang, X. Guo, C. Ye, N. Gao, Y. Fang, et al., "A Novel Multimodal MRI Analysis for Alzheimer's Disease Based on Convolutional Neural Network," in 2018 40th Annual International Conference of the IEEE Engineering in Medicine and Biology Society (EMBC), 2018, pp. 754-757. DOI: 10.1109/EMBC.2018.8512372

[78] K. Gunawardena, R. Rajapakse, and N. Kodikara, "Applying convolutional neural networks for pre-detection of alzheimer's disease from structural MRI data," in Mechatronics and Machine Vision in Practice (M2VIP), 2017 24th International Conference on, 2017, pp. 1-7. DOI: 10.1109/ M2VIP.2017.8211486

[79] G. A. Papakostas, A. Savio, M. Graña, and V. G. Kaburlasos, "A lattice computing approach to Alzheimer's disease computer assisted diagnosis based on MRI data," Neurocomputing, vol. 150, pp. 37-42, 2015. https:// doi.org/10.1016/j.neucom.2014.02.076

[80] F. Peng and Y. Ouyang, "Optimal clustering of railroad track maintenance jobs," Computer-Aided Civil and Infrastructure Engineering, vol. 29, pp. 235-247, 2014. https://doi.org/10.1111/mice.12036

[81] H. Wang, A. Yajima, R. Y. Liang*, and H. Castaneda, "Bayesian modeling of external corrosion in underground pipelines based on the integration of Markov chain Monte Carlo techniques and clustered inspection data," Computer-Aided Civil and Infrastructure Engineering, vol. 30, pp. 300316, 2015. https://doi.org/10.1111/mice.12096

[82] J. Huo, Y. Gao, W. Yang, and H. Yin, "Multi-instance dictionary learning for detecting abnormal events in surveillance videos," International journal of neural systems, vol. 24, p. 1430010, 2014. https://doi. org/10.1142/S0129065714300101

[83] T. Varghese, K. R. Sheela, P. Mathuranath, and A. Singh, "Evaluation of different stages of Alzheimer's disease using unsupervised clustering techniques and voxel based morphometry," in Information and Communication Technologies (WICT), 2012 World Congress on, 2012, pp. 953-958. DOI: 10.1109/WICT.2012.6409212

[84] K. Aderghal, A. Khvostikov, A. Krylov, J. Benois-Pineau, K. Afdel, and G. Catheline, "Classification of Alzheimer Disease on Imaging Modalities with Deep CNNs Using Cross-Modal Transfer Learning," in 2018 IEEE 31st International Symposium on Computer-Based Medical Systems (CBMS), 2018, pp. 345-350. DOI: 10.1109/CBMS.2018.00067

[85] S. Afzal, M. Maqsood, F. Nazir, U. Khan, F. Aadil, K. M. Awan, et al., "A Data Augmentation-Based Framework to Handle Class Imbalance Problem for Alzheimer's Stage Detection," IEEE Access, vol. 7, pp. 115528-115539, 2019. DOI: 10.1109/ACCESS.2019.2932786

[86] N. M. Khan, N. Abraham, and M. Hon, "Transfer Learning With Intelligent Training Data Selection for Prediction of Alzheimer's Disease," IEEE Access, vol. 7, 2019. DOI: 10.1109/ACCESS.2019.2920448

[87] M. Maqsood, F. Nazir, U. Khan, F. Aadil, H. Jamal, I. Mehmood, et al., "Transfer Learning Assisted Classification and Detection of Alzheimer's Disease Stages Using 3D MRI Scans," Sensors, vol. 19, 2019. https://doi. org $/ 10.3390 / \mathrm{s} 19112645$

[88] T. D. Phong, H. N. Duong, H. T. Nguyen, N. T. Trong, V. H. Nguyen, T. Van Hoa, et al., "Brain hemorrhage diagnosis by using deep learning," in Proceedings of the 2017 International Conference on
Machine Learning and Soft Computing, 2017, pp. 34-39. https://doi. org $/ 10.1145 / 3036290.3036326$

[89] S. Wang, Y. Shen, W. Chen, T. Xiao, and J. Hu, "Automatic recognition of mild cognitive impairment from mri images using expedited convolutional neural networks," in International Conference on Artificial Neural Networks, 2017, pp. 373-380. https://doi.org/10.1007/978-3-31968600-4_43.

[90] M. A. Nowrangi, C. G. Lyketsos, J.-M. S. Leoutsakos, K. Oishi, M. Albert, S. Mori, et al., "Longitudinal, region-specific course of diffusion tensor imaging measures in mild cognitive impairment and Alzheimer's disease," Alzheimer's \& Dementia, vol. 9, pp. 519-528, 2013. https://doi. org/10.1093/cercor/bhy031

[91] B. Cheng, M. Liu, D. Shen, Z. Li, D. Zhang, and A. s. D. N. Initiative, "Multi-domain transfer learning for early diagnosis of Alzheimer's disease," Neuroinformatics, vol. 15, pp. 115-132, 2017. https://doi. org/10.1007/s12021-016-9318-5

[92] T. Glozman, J. Solomon, F. Pestilli, and L. Guibas, "Shape-Attributes of Brain Structures as Biomarkers for Alzheimer's Disease," Journal of Alzheimer's Disease, vol. 56, pp. 287-295, 2017. DOI: 10.3233/JAD-160900

[93] M. Dyrba, M. Ewers, M. Wegrzyn, I. Kilimann, C. Plant, A. Oswald, et al., "Robust automated detection of microstructural white matter degeneration in Alzheimer's disease using machine learning classification of multicenter DTI data," PloS one, vol. 8, p. e64925, 2013. https://doi. org/10.1371/journal.pone.0064925

[94] W. Li, Y. Zhao, X. Chen, Y. Xiao, and Y. Qin, "Detecting Alzheimer's Disease on Small Dataset: A Knowledge Transfer Perspective," IEEE journal of biomedical and health informatics, 2018. DOI: 10.1109/ JBHI.2018.2839771

[95] T. Altaf, S. M. Anwar, N. Gul, M. N. Majeed, and M. Majid, "Multi-class Alzheimer's disease classification using image and clinical features," Biomedical Signal Processing and Control, vol. 43, pp. 64-74, 2018. https://doi.org/10.1016/j.bspc.2018.02.019

[96] O. B. Ahmed, M. Mizotin, J. Benois-Pineau, M. Allard, G. Catheline, C. B. Amar, et al., "Alzheimer's disease diagnosis on structural MR images using circular harmonic functions descriptors on hippocampus and posterior cingulate cortex," Computerized Medical Imaging and Graphics, vol. 44, pp. 13-25, 2015. https://doi.org/10.1016/j.eswa.2016.04.029

[97] D. Chitradevi and S. Prabha, "Analysis of brain sub regions using optimization techniques and deep learning method in Alzheimer disease," Applied Soft Computing, vol. 86, p. 105857, 2020. https://doi. org/10.1016/j.asoc.2019.105857

[98] X. Hao, Y. Bao, Y. Guo, M. Yu, D. Zhang, S. L. Risacher, et al., "Multimodal neuroimaging feature selection with consistent metric constraint for diagnosis of Alzheimer's disease," Medical Image Analysis, vol. 60, p. 101625, 2020. https://doi.org/10.1016/j.media.2019.101625

[99] C. Park, J. Ha, and S. Park, "Prediction of Alzheimer's disease based on deep neural network by integrating gene expression and DNA methylation dataset," Expert Systems with Applications, vol. 140, p. 112873, 2020. https://doi.org/10.1016/j.eswa.2019.112873

[100] A. Shikalgar and S. Sonavane, "Hybrid Deep Learning Approach for Classifying Alzheimer Disease Based on Multimodal Data," in Computing in Engineering and Technology, ed: Springer, 2020, pp. 511-520. https:// doi.org/10.1007/978-981-32-9515-5_49

[101] A. Giersch and J. T. Coull, "TRF1: It Was the Best of Time (s)...," Timing \& Time Perception, vol. 6, pp. 231-414, 2018. https://doi. org/10.1163/22134468-00603001

[102] S. Leandrou, S. Petroudi, P. A. Kyriacou, C. C. Reyes-Aldasoro, and C. S. Pattichis, "Quantitative MRI brain studies in mild cognitive impairment and Alzheimer's disease: a methodological review," IEEE reviews in biomedical engineering, vol. 11, pp. 97-111, 2018. DOI: 10.1109/ RBME.2018.2796598

[103] G. Litjens, T. Kooi, B. E. Bejnordi, A. A. A. Setio, F. Ciompi, M. Ghafoorian, et al., "A survey on deep learning in medical image analysis," Medical image analysis, vol. 42, pp. 60-88, 2017. https://doi.org/10.1016/j. media.2017.07.005

[104] D. Ravì, C. Wong, F. Deligianni, M. Berthelot, J. Andreu-Perez, B. Lo, et al., "Deep learning for health informatics," IEEE journal of biomedical and health informatics, vol. 21, pp. 4-21, 2016. DOI: 10.1109/ ICICI.2017.8365301

[105] P. V. Rouast, M. Adam, and R. Chiong, "Deep learning for human affect 
recognition: insights and new developments," IEEE Transactions on Affective Computing, 2019. DOI: 10.1109/TAFFC.2018.2890471

[106] C. R. Jack Jr, M. A. Bernstein, N. C. Fox, P. Thompson, G. Alexander, D. Harvey, et al., "The Alzheimer's disease neuroimaging initiative (ADNI): MRI methods," Journal of Magnetic Resonance Imaging: An Official Journal of the International Society for Magnetic Resonance in Medicine, vol. 27, pp. 685-691, 2008. https://doi.org/10.1002/jmri.21049

[107] K. A. Ellis, A. I. Bush, D. Darby, D. De Fazio, J. Foster, P. Hudson, et al., "The Australian Imaging, Biomarkers and Lifestyle (AIBL) study of aging: methodology and baseline characteristics of 1112 individuals recruited for a longitudinal study of Alzheimer's disease," International psychogeriatrics, vol. 21, pp. 672-687, 2009. https://doi.org/10.1017/ S1041610209009405

[108] D. S. Marcus, T. H. Wang, J. Parker, J. G. Csernansky, J. C. Morris, and R. L. Buckner, "Open Access Series of Imaging Studies (OASIS): crosssectional MRI data in young, middle aged, nondemented, and demented older adults," Journal of cognitive neuroscience, vol. 19, pp. 1498-1507, 2007. https://doi.org/10.1162/jocn.2007.19.9.1498

[109] A. Sedik, A. M. Iliyasu, A. El-Rahiem, M. E. Abdel Samea, A. AbdelRaheem, M. Hammad, et al., "Deploying Machine and Deep Learning Models for Efficient Data-Augmented Detection of COVID-19 Infections," Viruses, vol. 12, p. 769, 2020, https://doi.org/10.3390/v12070769

[110] A. Alghamdi, M. Hammad, H. Ugail, A. Abdel-Raheem, K. Muhammad, H. S. Khalifa, et al., "Detection of myocardial infarction based on novel deep transfer learning methods for urban healthcare in smart cities," Multimedia Tools and Applications, pp. 1-22, 2020, https://doi. org/10.1007/s11042-020-08769-x

[111] S. Toraman, T. B. Alakus, and I. Turkoglu, "Convolutional capsnet: A novel artificial neural network approach to detect COVID-19 disease from X-ray images using capsule networks," Chaos, Solitons \& Fractals, vol. 140, p. 110122, 2020, https://doi.org/10.1016/j.chaos.2020.110122

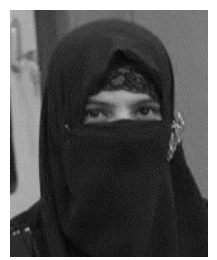

Sitara Afzal

Sitara Afzal has completed her MS from COMSATS University Islamabad, Attock campus. Her research interests are machine learning and image processing.

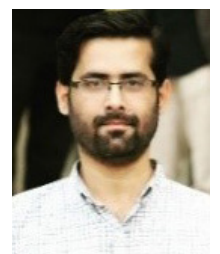

Muazzam Maqsood

Muazzam Maqsood is serving as an Assistant Professor at COMSATS University Islamabad, Attock Campus, Pakistan. He has earned his Ph.D. from UET Taxila in 2017. His research Interests includes machine learning, recommender systems, and image processing

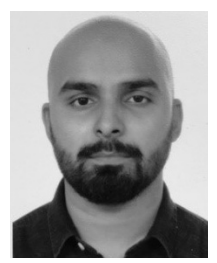

Umair Khan

Umair Khan is pursuing his Ph.D. from Italy. He has done his M.S. in Computer System Engineering from UET Peshawar, Pakistan. He is also a lecturer with COMSATS University Islamabad at Attock. His research concentrates on advanced concepts of image processing and machine learning.

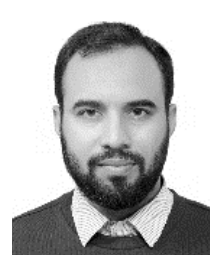

\section{Irfan Mehmood}

Irfan Mehmood is Assistant Professor of Applied Artificial Intelligence, Faculty of Engineering \& Informatics, School of Media, Design, and Technology, University of Bradford, UK. His areas of interest are multimedia analytics, information mining, and summarization. Specifically, he has made a significant contribution in the areas of visual surveillance, information mining, and data encryption.

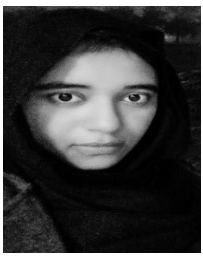

\section{Hina Nawaz}

Hina Nawaz is currently pursuing a master's degree with COMSATS University Islamabad, Attock campus. Her research interests are machine learning and image processing.

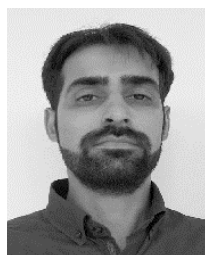

\section{Farhan Aadil}

Farhan Aadil received his B.S. degree in Computer Science from Allama Iqbal Open University, Pakistan in 2005. He pursued a career in computer science for 4 years (2005 to 2009). He received his M.S. degree in Software Engineering and the Ph.D. degree in Computer Engineering from the University of Engineering and Technology, Taxila, Pakistan in 2011 and 2016 respectively. He is currently working as an assistant professor at COMSATS University Islamabad, Attock Campus, Pakistan. His research interests include Machine Learning, Pattern recognition, and bio-inspired algorithms.

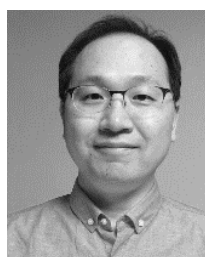

Oh-Young Song

Oh-Young Song is serving as an associate professor in the Department of Software at Sejong University in Korea. His research is in the area of computer graphics. $\mathrm{He}$ is particularly interested in physics-based animation, character animation, numerical algorithms, VR/AR, HCI, and machine learning.

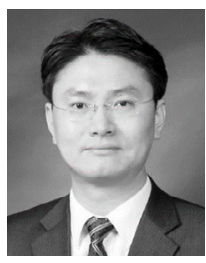

\section{Yunyoung Nam}

Yunyoung Nam received the B.S., M.S., and Ph.D. degrees in computer engineering from Ajou University, Korea in 2001, 2003, and 2007 respectively. He was a Senior Researcher with the Center of Excellence in Ubiquitous System, Stony Brook University, Stony Brook, NY, USA, from 2007 to 2010, where he was a Postdoctoral Researcher, from 2009 to 2013. He was a Research Professor with Ajou University, from 2010 to 2011. He was a Postdoctoral Fellow with the Worcester Polytechnic Institute, Worcester, MA, USA, from 2013 to 2014. He was the Director of the ICT Convergence Rehabilitation Engineering Research Center, Soonchunhyang University, from 2017 to 2020. He has been the Director of the ICT Convergence Research Center, Soonchunhyang University, since 2020, where he is currently an Assistant Professor with the Department of Computer Science and Engineering. His research interests include multimedia databases, ubiquitous computing, image processing, pattern recognition, context-awareness, conflict resolution, wearable computing, intelligent video surveillance, cloud computing, biomedical signal processing, rehabilitation, and healthcare systems. 